\title{
Condutas negativas à saúde em estudantes universitários brasileiros
}

\author{
Risk behaviors to health in Brazilian college students
}

Thiago Ferreira de Sousa ${ }^{1}$ Helma Pio Mororó José ${ }^{2}$ Aline Rodrigues Barbosa ${ }^{3}$
${ }^{1}$ Departamento de Ciências da Saúde, Reitoria, Universidade Estadual de Santa Cruz. Rodovia Ilhéus- Itabuna Km-16, Salobrinho. 45.662-900 Ilhéus BA Brasil. tfsousa_thiago@yahoo.com.br ${ }^{2}$ Departamento de Ciências Naturais, Universidade Estadual do Sudoeste da Bahia.

${ }^{3}$ Departamento de Educação Física, Universidade Federal de Santa Catarina.
Abstract The scope of this study was to estimate the prevalence and associated factors of lower levels of leisure-time physical activities, insufficient consumption of fruit and vegetables, alcohol consumption and smoking among college students from a public university in the northeastern region of Brazil. The sample was stratified in accordance with the academic program, period of study and year of admission to the university. The negative health-related behaviors were analyzed in relation to socio-demographic and program affiliation indicators by means of Prevalence Ratios. The most prevalent negative health-related behaviors were insufficient consumption of fruit and vegetables, lowers levels of leisure-time physical activities and alcohol consumption. Insufficient consumption of fruit, smoking and alcohol consumption were more prevalent among men. Furthermore, insufficient consumption of vegetables was higher among younger, unmarried, men after three years at the university, whose fathers failed to complete elementary school. Lower levels of leisure-time physical activities were higher among older women, who evaluated relationships with colleagues negatively and whose fathers failed to complete elementary school.

Key words Health behavior, Consumption of food, Consumption of alcohol, Physical activities, Students, Cross-sectional studies
Resumo O objetivo foi estimar as prevalências e os fatores associados aos menores níveis de atividades físicas no lazer, consumo insuficiente de frutas e de hortaliças, consumo de bebidas alcoólicas e hábito de fumar em estudantes de uma universidade pública da região Nordeste do Brasil. A amostra foi estratificada segundo o curso, o período de estudo e o ano de ingresso na universidade. As condutas negativas foram analisadas em relação aos indicadores sociodemográficos e de vínculo com a universidade, por meio das Razões de Prevalências. As condutas negativas de saúde mais prevalentes foram: consumo insuficiente de frutas, hortaliças, menores níveis de atividades físicas no lazer e consumo de bebidas alcoólicas. $O$ consumo insuficiente de frutas, hábito de fumar e consumo excessivo de bebidas alcoólicas foram maiores em homens. Além disso, o consumo insuficiente de hortaliças foi maior em homens, mais jovens, sem companheira, do pai com o ensino fundamental incompleto e com três anos de vivência na universidade. Os menores niveis de atividades físicas no lazer foram maiores em mulheres, mais velhas, com pai com ensino fundamental incompleto e que avaliaram negativamente os relacionamentos com os colegas.

Palavras-chave Condutas de saúde, Consumo de alimentos, Consumo de bebidas alcoólicas, Atividade motora, Estudantes, Estudos transversais 


\section{Introdução}

As condutas negativas de saúde, como a prática de atividades físicas em níveis insuficientes, consumo excessivo de bebidas alcoólicas e hábitos de fumar estão associadas com doenças crônicas não transmissíveis $(D C N T)^{1}$. Estes comportamentos de risco são responsáveis por grande parte do número de casos de morte em todo o mundo $^{1,2}$. No Brasil, em 2007, $72 \%$ de todas as mortes foram atribuídas às $\mathrm{DCNT}^{3}$.

Pesquisas realizadas com estudantes do ensino superior demonstraram que esse grupo estava predisposto a determinados comportamentos de risco à saúde durante o período acadêmi$\operatorname{co}^{4-9}$. O consumo de bebidas alcoólicas ${ }^{4,5}$, o hábito de fumar ${ }^{4}$, a alimentação inadequada ${ }^{6}$ e a inatividade física ${ }^{8}$ foram as condutas negativas de saúde com maiores prevalências entre os estudantes universitários.

No Brasil, estudos envolvendo de forma simultânea estudantes universitários de diferentes cursos ainda são escassos. Contudo, informações da Secretaria Nacional de Políticas sobre Drogas ${ }^{4}$ identificaram que o hábito de fumar e o consumo de bebidas alcoólicas foram mais prevalentes em homens e em estudantes do período noturno. Universitários da região Norte e Nordeste apresentaram maiores prevalências de uso, de pelo menos uma droga, como o tabaco ou bebidas alcoólicas na vida ${ }^{4}$. Estes dados evidenciam a necessidade de estudos sobre condutas negativas de saúde entre estudantes universitários das diferentes regiões do País.

O levantamento de informações sobre as condutas negativas de saúde de estudantes universitários das diferentes regiões do Brasil poderá incentivar políticas institucionais mais eficazes e direcionadas aos grupos mais expostos a hábitos negativos, pois, destaca-se o aumento do número de estudantes universitários nos últimos anos, especialmente na região Nordeste do Bra$\mathrm{sil}^{10}$. Sendo assim o objetivo deste estudo foi estimar as prevalências e os fatores associados aos menores níveis de atividades físicas no lazer, consumo insuficiente de frutas e de verduras, consumo excessivo de bebidas alcoólicas e o hábito de fumar em estudantes de uma universidade pública da região Nordeste do Brasil.

\section{Métodos}

Este estudo é de característica transversal, derivado do primeiro inquérito da pesquisa Monito- ramento dos Indicadores de Saúde e Qualidade de Vida em Acadêmicos (MONISA). O objetivo do Estudo MONISA é monitorar e caracterizar por um período de 10 anos os indicadores do estilo de vida e as condições ambientais e de aprendizagem em estudantes de uma universidade pública da região sul do Estado da Bahia, Brasil. O projeto do estudo foi aprovado pelo Comitê de Ética em Pesquisa da Universidade Estadual de Santa Cruz. Informações sobre os procedimentos metodológicos adotados no Estudo MONISA foram recentemente apresentados ${ }^{11}$ e serão descritos de forma breve.

A população deste estudo foram os 5.461 estudantes matriculados no $2^{\circ}$ semestre letivo de 2010 nos cursos presenciais de graduação da instituição. Não fizeram parte da população do estudo: estudantes aprovados no vestibular para o ingresso no segundo semestre letivo (calouros); estudantes dos cursos de ensino a distância e portadores de diploma de graduação, matriculados nos cursos de graduação presenciais. Os seguintes parâmetros foram considerados para as estimativas do tamanho amostral ${ }^{12}$ : população de referência, nível de confiança em $95 \%$, prevalência estimada em 50\%, erro amostral de 3 p.p. (pontos percentuais). Com base nesses parâmetros a amostra obtida foi de 893 estudantes, sendo acrescidos $20 \%$ para perdas e recusas e $15 \%$ para o controle de fatores de confusão nas análises ajustadas, perfazendo uma amostra de 1.232 estudantes.

A amostra deste estudo pôde detectar com poder de $80 \%$ e nível de confiança de $95 \%$, razões de prevalências significativas, nas análises brutas, para os menores níveis de atividades físicas no lazer, acima de 1,2 e abaixo de 0,8 ; consumo insuficiente de frutas, acima de 1,1 e abaixo de 0,9 ; consumo insuficiente de verduras, acima de 1,1 e abaixo de 0,9 ; consumo excessivo de bebidas alcoólicas, acima de 1,2 e abaixo de 0,8; e fumantes, acima de 4,4 e abaixo de 0,2 .

A amostra foi estratificada e proporcional de acordo com os 30 cursos da instituição; período de estudo (diurno e noturno); e, anos de ingresso na universidade $(2010,2009,2008$ e 2007 e anterior). Os estudantes foram selecionados em cada estrato (seleção aleatória simples), mediante a lista de matrícula em ordem alfabética e foram procurados em até três tentativas, em dias e horários diferentes. Aqueles que não foram encontrados ou não tiveram interesse em participar foram considerados como perdas e recusas, respectivamente, não havendo reposição.

A coleta de dados (setembro a novembro de 2010) foi realizada nas salas de aula da universi- 
dade, antes, durante, ou ao final das aulas (conforme consentimento dos estudantes selecionados e professores que estavam em sala no momento da coleta). As informações foram obtidas pelo questionário Isaq-A (Indicadores de Saúde e Qualidade de Vida de Acadêmicos) que foi previamente testado, nos meses de julho e agosto, quanto à validade de face e conteúdo; clareza $\mathrm{e}$ pré-testagem; e, avaliação dos níveis de reprodutibilidade com intervalo de uma semana ${ }^{13}$.

\section{Variáveis desfechos}

As variáveis desfechos foram dicotomizadas e as categorias relacionadas ao riscos à saúde foram: menor nivel de atividade física no lazer, ou seja, $<500$ MET.min $/ \mathrm{sem}^{14}$ (MET.min $/ \mathrm{sem}=$ unidade empregada para estimar o custo metabólico, em consumo de oxigênio, relativo a prática de atividade física em uma semana, considerando 1 MET igual a 3,5 ml.kg.min de oxigênio em repouso) em atividades físicas no lazer, obtidos mediante a multiplicação da quantidade de dias de prática pelo tempo médio em minutos de prática por dia, posteriormente multiplicados pelo equivalente metabólico (MET) relativo à atividade física praticada, com base no compêndio de Ainsworth et al. ${ }^{15}$; consumo insuficiente de frutas, até quatro dias por semana ${ }^{16}$; consumo insuficiente de hortaliças, até quatro dias por semana ${ }^{16}$; consumo excessivo de bebidas alcoólicas, consumir nos últimos trinta dias, cinco ou mais doses de bebidas alcoólicas em uma mesma ocasião, sendo a dose considerada como: meia garrafa de cerveja, ou uma lata de cerveja, ou um copo de vinho ou uma dose de uísque, conhaque, cachaça ou vodka ${ }^{17}$; fumantes, independente da quantidade e frequência de consumo ${ }^{18}$.

\section{Variáveis exploratórias}

As variáveis exploratórias foram: sexo (masculino e feminino); faixa de idade (17-19, 20-21, $22-24$ e $\geq 25$ anos); situação conjugal (sem companheiro e com companheiro); escolaridade do pai, (ensino fundamental incompleto, ensino fundamental completo, ensino médio completo e ensino superior completo); escolaridade da mãe (ensino fundamental incompleto, ensino fundamental completo, ensino médio completo e ensino superior completo); renda pessoal (em reais de acordo com salário mínimo vigente em 2010 de $\mathrm{R} \$ 510,00$, categorizado em não tem, $<\mathrm{R} \$$ 510,00 e $\geq R \$ 510,00$ ); período de estudo (diurno e noturno); ano de ingresso na universidade (2010,
2009, 2008 e 2007 e anterior); autoavaliação dos relacionamentos com os colegas do curso (positiva e negativa).

A tabulação foi realizada no programa EpiData 3.1 e as análises estatísticas no Programa SPSS, versão 16.0. Foi utilizada estatística descritiva (frequências absoluta e relativa, média, mediana, desvio padrão (DP), valores mínimo e máximo e, intervalos de confiança) e as Razões de Prevalências (RP), nas análises brutas e ajustadas, como medida de associação, estimadas pela regressão de Poisson, com ajuste para a variância robusta.

Todas as variáveis exploratórias foram para análise ajustada, independente do valor de $p$ da análise bruta. As variáveis foram ajustadas, de acordo com o seguinte modelo hierárquico: $1^{\circ}$ nível, sexo e faixa de idade; $2^{\circ}$ nível, situação conjugal, renda pessoal em reais, escolaridade do pai e da mãe; $3^{\circ}$ nível, período de estudo, ano de ingresso na universidade e autoavaliação dos relacionamentos com os colegas de curso. As variáveis que apresentaram valor de $\mathrm{p}$ do teste de Wald $<0,20$ foram ajustadas no mesmo nível e seguiram para os níveis superiores, mediante o procedimento de seleção de variáveis backward. O nível de significância adotado foi de 5\%.

\section{Resultados}

Da amostra de 1.232 estudantes selecionados, participaram deste estudo $88 \%(n=1.084)$, sendo uma participação superior a $70 \%$ em todos os cursos da instituição. Não foram observadas diferenças entre a amostra alcançada e as perdas/recusas (12\%), em relação ao sexo, período de estudo e características da população de estudo (dados não apresentados).

A média etária dos participantes foi 23,5 anos ( $\mathrm{DP}=5,2 ; 17$ a 52 anos) e a mediana 22 anos. Houve predominância de estudantes do sexo feminino $(54,7 \%)$ e $29,2 \%$ eram da faixa etária 22 24 anos, $86,4 \%$ sem companheiro, com renda pessoal $\geq R \$ 510,00$ (35,9\%), com pais e mães com nível de escolaridade referente ao ensino médio completo e que autoavaliaram os relacionamentos com os colegas do seu curso como positivo $(90,7 \%)$ (Tabela 1$)$. A maioria dos estudantes apresentou menor nível de atividade física no lazer $(54,1 \%)$ e consumo insuficiente de frutas e de hortaliças foi observado para $81,2 \%$ e $57,0 \%$, respectivamente; a prevalência de consumo de bebidas alcoólicas em $41,3 \%$ e o hábito de fumar para apenas 3,1\% (Tabela 2). 
Tabela 1. Características sociodemográficas e de vínculo com a universidade em estudantes de uma instituição pública do Nordeste do Brasil. MONISA 2010.

\begin{tabular}{|c|c|c|c|}
\hline Variáveis & $\mathbf{n}$ & $\%$ & IC95\% \\
\hline \multicolumn{4}{|l|}{ Sexo } \\
\hline Masculino & 491 & 45,3 & $42,4-48,3$ \\
\hline Feminino & 592 & 54,7 & $51,7-57,6$ \\
\hline \multicolumn{4}{|l|}{ Faixa de idade } \\
\hline 17 a 19 anos & 186 & 17,4 & $15,1-19,7$ \\
\hline 20 e 21 anos & 270 & 25,3 & $22,6-27,9$ \\
\hline 22 a 24 anos & 312 & 29,2 & $26,4-31,9$ \\
\hline 25 a 52 anos & 301 & 28,1 & $25,4-30,8$ \\
\hline \multicolumn{4}{|l|}{ Situação conjugal } \\
\hline Sem companheiro(a) & 937 & 86,4 & $84,4-88,5$ \\
\hline Com companheiro(a) & 147 & 13,6 & $11,5-15,6$ \\
\hline \multicolumn{4}{|l|}{ Renda pessoal em reais } \\
\hline Não tem & 348 & 33,2 & $30,3-36,1$ \\
\hline$<\mathrm{R} \$ 510,00$ & 324 & 30,9 & $28,1-33,7$ \\
\hline$\geq \mathrm{R} \$ 510,00$ & 376 & 35,9 & $33,0-38,8$ \\
\hline \multicolumn{4}{|l|}{ Escolaridade do pai } \\
\hline Ensino fundamental incompleto & 300 & 29,9 & $27,1-32,7$ \\
\hline Ensino fundamental completo & 142 & 14,2 & $12,0-16,3$ \\
\hline Ensino médio completo & 410 & 40,9 & $37,8-43,9$ \\
\hline Ensino superior completo & 151 & 15,0 & $12,8-17,2$ \\
\hline \multicolumn{4}{|l|}{ Escolaridade da mãe } \\
\hline Ensino fundamental incompleto & 234 & 22,6 & $20,0-25,2$ \\
\hline Ensino fundamental completo & 114 & 11,0 & $9,1-12,9$ \\
\hline Ensino médio completo & 477 & 46,1 & $43,0-49,1$ \\
\hline Ensino superior completo & 210 & 20,3 & $17,8-22,7$ \\
\hline \multicolumn{4}{|l|}{ Período de estudo } \\
\hline Diurno & 735 & 67,8 & $65,0-70,6$ \\
\hline Noturno & 349 & 32,2 & $29,4-35,0$ \\
\hline \multicolumn{4}{|l|}{ Ano de ingresso } \\
\hline 2010 & 233 & 21,5 & $19,0-23,9$ \\
\hline 2009 & 267 & 24,6 & $22,1-27,2$ \\
\hline 2008 & 225 & 20,8 & $18,3-23,2$ \\
\hline 2007 e anterior & 359 & 33,1 & $30,3-35,9$ \\
\hline \multicolumn{4}{|c|}{ Auto-avaliação dos relacionamentos com os colegas } \\
\hline Positiva & 976 & 90,7 & $89,0-92,4$ \\
\hline Negativa & 100 & 9,3 & $7,5-11,0$ \\
\hline
\end{tabular}

$\%=$ Prevalência; IC95\% = Intervalo de Confiança a 95\%.

A análise bruta (Tabela 3) demonstrou que as seguintes características foram associadas às prevalências de menores níveis de atividades físicas no lazer: mulheres (RP: 1,89; IC95\%: 1,662,16), estudantes com companheiro (RP: 1,18; IC95\%: 1,02-1,36), com pai e mãe com escolaridade referente ao ensino fundamental incompleto e que autoavaliaram os relacionamentos com os colegas como negativo (RP: 1,21; IC95\%: 1,03$1,42)$. O consumo insuficiente de frutas foi mais prevalente entre os homens e o consumo insuficiente de hortaliças foi maior em homens, me- nor grupo etário, estudantes sem companheiro e que relataram não ter renda. A maior prevalência de consumo de bebidas alcoólicas foi observada entre homens e estudantes com pai e mãe com ensino médio e superior completo. As mulheres apresentaram menor prevalência de hábito de fumar que os homens.

Nas análises ajustadas (Tabela 4) os fatores que se mantiveram associados com maiores prevalências de menores níveis de atividades físicas no lazer foram: as mulheres ( $\mathrm{RP}=1,92$; IC95\% $=$ 1,68-2,19), estudantes da faixa etária mais velha 
Tabela 2. Prevalências das condutas negativas à saúde de acordo com variáveis sociodemográficas e de vínculo com a universidade em estudantes de uma instituição pública do Nordeste do Brasil. MONISA 2010.

\begin{tabular}{|c|c|c|c|c|c|c|}
\hline \multirow[t]{2}{*}{ Variáveis } & \multicolumn{2}{|c|}{$\begin{array}{l}\text { Menores níveis } \\
\text { de atividades } \\
\text { físicas no lazer }\end{array}$} & \multicolumn{2}{|c|}{$\begin{array}{l}\text { Consumo } \\
\text { insuficiente } \\
\text { de frutas }\end{array}$} & \multicolumn{2}{|c|}{$\begin{array}{c}\text { Consumo } \\
\text { insuficiente } \\
\text { de hortaliças }\end{array}$} \\
\hline & $\mathbf{n}$ & $\%$ & $\mathbf{n}$ & $\%$ & $\mathbf{n}$ & $\%$ \\
\hline Total & 1.040 & 54,1 & 1.062 & 81,2 & 1.057 & 57,0 \\
\hline \multicolumn{7}{|l|}{ Sexo } \\
\hline Masculino & 468 & 36,3 & 485 & 84,5 & 482 & 61,0 \\
\hline Feminino & 571 & 68,8 & 576 & 78,3 & 574 & 53,5 \\
\hline \multicolumn{7}{|l|}{ Faixa de idade } \\
\hline 17 a 19 anos & 180 & 48,3 & 182 & 82,4 & 184 & 56,5 \\
\hline 20 e 21 anos & 261 & 56,7 & 264 & 85,2 & 267 & 64,4 \\
\hline 22 a 24 anos & 304 & 51,6 & 308 & 79,2 & 305 & 59,7 \\
\hline 25 a 52 anos & 281 & 57,3 & 294 & 78,6 & 286 & 46,8 \\
\hline \multicolumn{7}{|l|}{ Situação conjugal } \\
\hline Sem companheiro(a) & 904 & 52,8 & 919 & 81,5 & 915 & 59,3 \\
\hline Com companheiro(a) & 136 & 62,5 & 143 & 79,0 & 142 & 41,5 \\
\hline \multicolumn{7}{|l|}{ Renda pessoal em reais } \\
\hline Não tem & 332 & 52,1 & 340 & 84,4 & 343 & 63,6 \\
\hline$<\mathrm{R} \$ 510,00$ & 313 & 57,8 & 317 & 80,1 & 316 & 54,7 \\
\hline$\geq \mathrm{R} \$ 510,00$ & 361 & 52,1 & 370 & 78,9 & 363 & 53,2 \\
\hline \multicolumn{7}{|l|}{ Escolaridade do pai } \\
\hline Ensino fundamental incompleto & 286 & 63,6 & 291 & 79,7 & 286 & 59,8 \\
\hline Ensino fundamental completo & 138 & 53,6 & 142 & 85,2 & 137 & 56,9 \\
\hline Ensino médio completo & 395 & 49,9 & 398 & 80,9 & 407 & 59,2 \\
\hline Ensino superior completo & 144 & 42,4 & 151 & 79,5 & 151 & 52,2 \\
\hline \multicolumn{7}{|l|}{ Escolaridade da mãe } \\
\hline Ensino fundamental incompleto & 220 & 63,2 & 231 & 80,1 & 222 & 56,8 \\
\hline Ensino fundamental completo & 112 & 61,6 & 112 & 83,0 & 111 & 54,0 \\
\hline Ensino médio completo & 458 & 52,2 & 464 & 80,6 & 469 & 56,7 \\
\hline Ensino superior completo & 205 & 42,4 & 207 & 81,2 & 208 & 61,5 \\
\hline \multicolumn{7}{|l|}{ Período de estudo } \\
\hline Diurno & 706 & 52,3 & 717 & 82,4 & 719 & 59,0 \\
\hline Noturno & 334 & 58,1 & 345 & 78,6 & 338 & 52,7 \\
\hline \multicolumn{7}{|l|}{ Ano de ingresso } \\
\hline 2010 & 223 & 53,4 & 227 & 82,4 & 227 & 55,9 \\
\hline 2009 & 254 & 55,1 & 264 & 80,7 & 261 & 52,1 \\
\hline 2008 & 219 & 56,1 & 222 & 82,4 & 218 & 64,2 \\
\hline 2007 e anterior & 344 & 52,6 & 349 & 79,9 & 351 & 56,7 \\
\hline \multicolumn{7}{|c|}{ Auto-avaliação dos relacionamentos com os colegas } \\
\hline Positiva & 938 & 53,1 & 956 & 80,9 & 952 & 56,9 \\
\hline Negativa & 98 & 64,3 & 99 & 83,8 & 97 & 57,7 \\
\hline
\end{tabular}

e que autoavaliaram os relacionamentos com os colegas do curso como negativo; e, com menores prevalências, estudantes com pai com ensino superior completo $(\mathrm{RP}=0,72 ; \mathrm{IC} 95 \%=0,57-0,91)$. As mulheres apresentaram menores prevalências de consumo insuficiente de frutas e de hortaliças; além disso, menores prevalências de consumo insuficiente de hortaliças foram observadas em estudantes da faixa etária mais velha (25-
52 anos), com companheiro, com pai com escolaridade referente ao superior completo e com maior prevalência os estudantes com três anos de vivência acadêmica (ano de ingresso 2008). As mulheres apresentaram menor prevalência de consumo de bebidas alcoólicas ( $\mathrm{RP}=0,63$; IC95\% $=0,55-0,73)$ e hábito de fumar $(\mathrm{RP}=0,20$; IC95\% $=0,08-0,47)$ quando comparadas aos homens. Os estudantes que ingressaram na universidade 
Tabela 2. continuação

\begin{tabular}{|c|c|c|c|c|}
\hline \multirow{2}{*}{ Variáveis } & \multicolumn{2}{|c|}{$\begin{array}{c}\text { Consumo } \\
\text { de bebidas alcoólicas }\end{array}$} & \multicolumn{2}{|c|}{ Fumantes } \\
\hline & $\mathbf{n}$ & $\%$ & $\mathbf{n}$ & $\%$ \\
\hline Total & 1.073 & 41,3 & 1.079 & 3,1 \\
\hline \multicolumn{5}{|l|}{ Sexo } \\
\hline Masculino & 480 & 51,9 & 488 & 5.3 \\
\hline Feminino & 592 & 32,8 & 590 & 1.2 \\
\hline \multicolumn{5}{|l|}{ Faixa de idade } \\
\hline 17 a 19 anos & 184 & 37,5 & 185 & 1,6 \\
\hline 20 e 21 anos & 266 & 37,6 & 270 & 2,2 \\
\hline 22 a 24 anos & 309 & 43,4 & 309 & 2,9 \\
\hline 25 a 52 anos & 299 & 44,5 & 300 & 4,7 \\
\hline \multicolumn{5}{|l|}{ Situação conjugal } \\
\hline Sem companheiro(a) & 928 & 41,6 & 932 & 2,9 \\
\hline Com companheiro(a) & 145 & 39,3 & 147 & 4,1 \\
\hline \multicolumn{5}{|l|}{ Renda pessoal em reais } \\
\hline Não tem & 346 & 41,0 & 345 & 2,3 \\
\hline$<\mathrm{R} \$ 510,00$ & 320 & 35,3 & 323 & 3,1 \\
\hline$\geq \mathrm{R} \$ 510,00$ & 371 & 46,9 & 375 & 3,7 \\
\hline \multicolumn{5}{|l|}{ Escolaridade do pai } \\
\hline Ensino fundamental incompleto & 298 & 35,6 & 299 & 2,0 \\
\hline Ensino fundamental completo & 139 & 41,0 & 141 & 2,1 \\
\hline Ensino médio completo & 405 & 43,7 & 409 & 3,2 \\
\hline Ensino superior completo & 151 & 49,0 & 150 & 4,0 \\
\hline \multicolumn{5}{|l|}{ Escolaridade da mãe } \\
\hline Ensino fundamental incompleto & 231 & 34,6 & 233 & 1,7 \\
\hline Ensino fundamental completo & 112 & 33,0 & 114 & 2,6 \\
\hline Ensino médio completo & 472 & 43,2 & 474 & 2,7 \\
\hline Ensino superior completo & 210 & 48,1 & 210 & 4,3 \\
\hline \multicolumn{5}{|l|}{ Período de estudo } \\
\hline Diurno & 728 & 42,2 & 731 & 3,3 \\
\hline Noturno & 345 & 39,4 & 348 & 2,6 \\
\hline \multicolumn{5}{|l|}{ Ano de ingresso } \\
\hline 2010 & 232 & 42,7 & 233 & 4,3 \\
\hline 2009 & 263 & 31,2 & 265 & 2,3 \\
\hline 2008 & 222 & 36,9 & 223 & 2,2 \\
\hline 2007 e anterior & 356 & 50,6 & 358 & 3,3 \\
\hline \multicolumn{5}{|c|}{ Auto-avaliação dos relacionamentos com os colegas } \\
\hline Positiva & 966 & 41,7 & 975 & 2,9 \\
\hline Negativa & 100 & 38,0 & 97 & 5,2 \\
\hline
\end{tabular}

$\%=$ Prevalência .

em 2008 apresentaram menor prevalência do hábito de fumar que os ingressantes em 2010 (RP $=0,26$; IC $95 \%=0,07-0,96)$.

\section{Discussão}

Os resultados mostraram que o consumo insuficiente de frutas e hortaliças foram os comportamentos de risco mais prevalentes entre os estudantes, similar ao observado em estudantes de uma universidade do Estado de Pernambuco ${ }^{19}$ e de países como Colômbia ${ }^{6}$ e Egito ${ }^{20}$. O consumo insuficiente de frutas e hortaliças foi mais prevalente entre os homens, comparado às mulheres, podendo indicar maior preocupação alimentar por parte do grupo feminino, no entanto, essas diferenças não foram observadas em outras pesquisas com estudantes universitários ${ }^{7,19}$. Além disso, os estudantes universitários mais jovens apresentaram maiores prevalências de consumo insuficiente de hortaliças. Possivelmente, os va- 
Tabela 3. Análise bruta entre condutas negativas à saúde e variáveis sociodemográficas e de vínculo com a universidade em estudantes de uma instituição pública do Nordeste do Brasil. MONISA 2010.

\begin{tabular}{|c|c|c|c|c|c|c|}
\hline \multirow[t]{2}{*}{ Variáveis } & \multicolumn{2}{|c|}{$\begin{array}{l}\text { Menores níveis de } \\
\text { atividades físicas no lazer }\end{array}$} & \multicolumn{2}{|c|}{$\begin{array}{c}\text { Consumo insuficiente } \\
\text { de frutas }\end{array}$} & \multicolumn{2}{|c|}{$\begin{array}{c}\text { Consumo insuficiente } \\
\text { de hortaliças }\end{array}$} \\
\hline & RP (IC95\%) & p & RP (IC95\%) & $\mathbf{p}$ & RP (IC95\%) & $\mathrm{p}$ \\
\hline Sexo & & $<0,001$ & & 0,009 & & 0,01 \\
\hline Masculino & 1,00 & & 1,00 & & 1,00 & \\
\hline Feminino & $1,89(1,66-2,16)$ & & $0,93(0,87-0,98)$ & & $0,88(0,79-0,97)$ & \\
\hline Faixa de idade & & $0,19^{*}$ & & $0,09^{*}$ & & $0,005^{*}$ \\
\hline 17 a 19 anos & 1,00 & & 1,00 & & 1,00 & \\
\hline 20 e 21 anos & $1,17(0,98-1,41)$ & & $1,03(0,95-1,12)$ & & $1,14(0,98-1,33)$ & \\
\hline 22 a 24 anos & $1,07(0,89-1,29)$ & & $0,96(0,88-1,05)$ & & $1,06(0,90-1,23)$ & \\
\hline 25 a 52 anos & $1,18(0,99-1,42)$ & & $0,95(0,87-1,04)$ & & $0,83(0,69-0,99)$ & \\
\hline Situação conjugal & & 0,02 & & 0,50 & & 0,001 \\
\hline Sem companheiro(a) & 1,00 & & 1,00 & & 1,00 & \\
\hline Com companheiro(a) & $1,18(1,02-1,36)$ & & $0,97(0,89-1,06)$ & & $0,70(0,57-0,86)$ & \\
\hline Renda pessoal em reais & & $0,96^{*}$ & & $0,06^{*}$ & & $0,005^{*}$ \\
\hline Não tem & 1,00 & & 1,00 & & 1,00 & \\
\hline$<\mathrm{R} \$ 510,00$ & $1,11(0,96-1,28)$ & & $0,95(0,88-1,02)$ & & $0,86(0,76-0,98)$ & \\
\hline$\geq \mathrm{R} \$ 510,00$ & $1,00(0,87-1,15)$ & & $0,93(0,87-1,00)$ & & $0,84(0,74-0,95)$ & \\
\hline Escolaridade do pai & & $<0,001^{*}$ & & $0,93^{*}$ & & $0,29^{*}$ \\
\hline Ensino fundamental incompleto & 1,00 & & 1,00 & & 1,00 & \\
\hline Ensino fundamental completo & $0,84(0,70-1,01)$ & & $1,07(0,98-1,17)$ & & $0,95(0,80-1,13)$ & \\
\hline Ensino médio completo & $0,78(0,69-0,89)$ & & $1,01(0,94-1,09)$ & & $0,99(0,87-1,12)$ & \\
\hline Ensino superior completo & $0,66(0,54-0,82)$ & & $1,00(0,90-1,10)$ & & $0,87(0,73-1,05)$ & \\
\hline Escolaridade da mãe & & $<0,001^{*}$ & & $0,89^{*}$ & & $0,35^{*}$ \\
\hline Ensino fundamental incompleto & 1,00 & & 1,00 & & 1,00 & \\
\hline Ensino fundamental completo & $0,97(0,81-1,16)$ & & $1,04(0,93-1,15)$ & & $0,95(0,77-1,17)$ & \\
\hline Ensino médio completo & $0,82(0,72-0,94)$ & & $1,01(0,93-1,09)$ & & $1,00(0,87-1,15)$ & \\
\hline Ensino superior completo & $0,67(0,56-0,81)$ & & $1,01(0,92-1,11)$ & & $1,08(0,93-1,27)$ & \\
\hline Período de estudo & & 0,07 & & 0,14 & & 0,06 \\
\hline Diurno & 1,00 & & 1,00 & & 1,00 & \\
\hline Noturno & $1,11(0,99-1,25)$ & & $0,95(0,89-1,02)$ & & $0,89(0,79-1,00)$ & \\
\hline Ano de ingresso & & $0,82^{*}$ & & $0,56^{*}$ & & $0,38^{*}$ \\
\hline 2010 & 1,00 & & 1,00 & & 1,00 & \\
\hline 2009 & $1,03(0,87-1,22)$ & & $0,98(0,90-1,06)$ & & $0,93(0,79-1,10)$ & \\
\hline 2008 & $1,05(0,89-1,25)$ & & $1,00(0,92-1,09)$ & & $1,15(0,98-1,34)$ & \\
\hline 2007 e anterior & $0,99(0,84-1,15)$ & & $0,97(0,89-1,05)$ & & $1,01(0,87-1,17)$ & \\
\hline $\begin{array}{l}\text { Auto-avaliação dos relacionamentos } \\
\text { com os colegas }\end{array}$ & & 0,02 & & 0,44 & & 0,88 \\
\hline Positiva & 1,00 & & 1,00 & & 1,00 & \\
\hline Negativa & $1,21(1,03-1,42)$ & & $1,04(0,95-1,14)$ & & $1,01(0,85-1,21)$ & \\
\hline
\end{tabular}

lores culturais em relação ao consumo desses alimentos sejam mais marcantes em adultos com mais idade e maior escolaridade ${ }^{16,21}$. Vale salientar que um padrão alimentar composto pelo consumo de frutas e hortaliças, tende a favorecer menores riscos de morbidades e mortalidade cardiovascular $^{22}$.

Outro importante comportamento de risco à saúde é o baixo nível de atividades físicas ${ }^{23}$. Ten- do em vista a relevância da prática de atividades físicas no lazer em estudantes universitários, observou-se neste estudo que sua prevalência foi maior do que as verificadas em estudantes universitários brasileiros ${ }^{9,24,25}$ e de diferentes países com cultura e desenvolvimento econômico diversificado ${ }^{26}$. Os menores níveis de atividades físicas estariam associados à ausência de locais adequados para a prática ${ }^{27}$, ou mesmo à falta de 
Tabela 3. continuação

\begin{tabular}{|c|c|c|c|c|}
\hline \multirow[t]{2}{*}{ Variáveis } & \multicolumn{2}{|c|}{$\begin{array}{c}\text { Consumo de } \\
\text { bebidas alcoólicas }\end{array}$} & \multicolumn{2}{|c|}{ Fumantes } \\
\hline & RP (IC95\%) & $\mathrm{p}$ & RP (IC95\%) & p \\
\hline Sexo & & $<0,001$ & & $<0,001$ \\
\hline Masculino & 1,00 & & 1,00 & \\
\hline Feminino & $0,63(0,55-0,73)$ & & $0,22(0,09-0,51)$ & \\
\hline Faixa de idade & & $0,05^{\star}$ & & $0,05^{\star}$ \\
\hline 17 a 19 anos & 1,00 & & 1,00 & \\
\hline 20 e 21 anos & $1,00(0,79-1,28)$ & & $1,37(0,35-5,41)$ & \\
\hline 22 a 24 anos & $1,16(0,92-1,45)$ & & $1,80(0,49-6,55)$ & \\
\hline 25 a 52 anos & $1,19(0,95-1,49)$ & & $2,88(0,84-9,88)$ & \\
\hline Situação conjugal & & 0,61 & & 0,44 \\
\hline Sem companheiro(a) & 1,00 & & 1,00 & \\
\hline Com companheiro(a) & $0,94(0,76-1,17)$ & & $1,41(0,59-3,35)$ & \\
\hline Renda pessoal em reais & & $0,10^{*}$ & & $0,27^{\star}$ \\
\hline Não tem & 1,00 & & 1,00 & \\
\hline$<\mathrm{R} \$ 510,00$ & $0,86(0,71-1,04)$ & & $1,33(0,53-3,34)$ & \\
\hline$\geq \mathrm{R} \$ 510,00$ & $1,14(0,97-1,35)$ & & $1,61(0,68-3,79)$ & \\
\hline Escolaridade do pai & & $0,004^{*}$ & & $0,18^{*}$ \\
\hline Ensino fundamental incompleto & 1,00 & & 1,00 & \\
\hline Ensino fundamental completo & $1,15(0,90-1,48)$ & & $1,06(0,27-4,18)$ & \\
\hline Ensino médio completo & $1,23(1,02-1,48)$ & & $1,58(0,61-4,12)$ & \\
\hline Ensino superior completo & $1,38(1,10-1,72)$ & & $1,99(0,65-6,08)$ & \\
\hline Escolaridade da mãe & & $0,001^{*}$ & & $0,14^{*}$ \\
\hline Ensino fundamental incompleto & 1,00 & & 1,00 & \\
\hline Ensino fundamental completo & $0,95(0,69-1,31)$ & & $1,53(0,35-6,73)$ & \\
\hline Ensino médio completo & $1,25(1,02-1,53)$ & & $1,60(0,53-4,84)$ & \\
\hline Ensino superior completo & $1,39(1,11-1,74)$ & & $2,50(0,78-7,99)$ & \\
\hline Período de estudo & & 0,40 & & 0,54 \\
\hline Diurno & 1,00 & & 1,00 & \\
\hline Noturno & $0,93(0,80-1,09)$ & & $0,79(0,37-1,68)$ & \\
\hline Ano de ingresso & & $0,005^{*}$ & & $0,71^{*}$ \\
\hline 2010 & 1,00 & & 1,00 & \\
\hline 2009 & $0,73(0,58-0,92)$ & & $0,53(0,19-1,43)$ & \\
\hline 2008 & $0,86(0,69-1,09)$ & & $0,52(0,18-1,50)$ & \\
\hline 2007 e anterior & $1,18(0,99-1,42)$ & & $0,78(0,34-1,78)$ & \\
\hline $\begin{array}{l}\text { Auto-avaliação dos relacionamentos } \\
\text { com os colegas }\end{array}$ & & 0,48 & & 0,22 \\
\hline Positiva & 1,00 & & 1,00 & \\
\hline Negativa & $0,91(0,70-1,18)$ & & $1,79(0,71-4,54)$ & \\
\hline
\end{tabular}

RP = Razões de Prevalências; IC95\% = Intervalo de Confiança a 95\%; ${ }^{*}$ Teste de Wald para tendência linear.

organização do tempo destinado às atividades acadêmicas e lazer. As mulheres e os estudantes mais velhos apresentaram maior prevalência de menores níveis de atividades físicas no lazer, assim como o identificado em outros estudos ${ }^{9,24,25}$. Possivelmente, estudantes mais velhos estão mais envolvidos em atividades ocupacionais e que juntamente com as obrigações acadêmicas tendem a se envolver menos em atividades físicas no lazer $^{28}$. Quanto ao sexo, aspectos culturais podem estar relacionados às maiores prevalências de inatividade física no lazer em mulheres, tendo em vista o estímulo a práticas menos ativas no lazer e com menor intensidade.

Os resultados mostraram que os estudantes universitários, que informaram a escolaridade do pai como ensino fundamental incompleto apresentaram maior prevalência de menor nível de atividade física no lazer. Em estudantes universitários sergipanos essa associação foi observada, no en- 
Tabela 4. Análise ajustada ${ }^{\ddagger}$ entre condutas negativas à saúde e variáveis sociodemográficas e de vínculo com a universidade em estudantes de uma instituição pública do Nordeste do Brasil. MONISA 2010.

\begin{tabular}{|c|c|c|c|c|c|c|}
\hline \multirow[t]{2}{*}{ Variáveis } & \multicolumn{2}{|c|}{$\begin{array}{l}\text { Menores níveis de } \\
\text { atividades físicas no lazer }\end{array}$} & \multicolumn{2}{|c|}{$\begin{array}{c}\text { Consumo insuficiente } \\
\text { de frutas }\end{array}$} & \multicolumn{2}{|c|}{$\begin{array}{c}\text { Consumo insuficiente } \\
\text { de hortaliças }\end{array}$} \\
\hline & RP (IC95\%) & $\mathbf{p}$ & RP (IC95\%) & $\mathbf{p}$ & RP (IC95\%) & $\mathbf{p}$ \\
\hline $\mathrm{Sexo}^{\mathrm{a}}$ & & $<0,001$ & & 0,01 & & 0,009 \\
\hline Masculino & 1,00 & & 1,00 & & 1,00 & \\
\hline Feminino & $1,92(1,68-2,19)$ & & $0,93(0,87-0,98)$ & & $0,87(0,78-0,97)$ & \\
\hline Faixa de idade ${ }^{a}$ & & $0,08^{*}$ & & $0,07^{*}$ & & $0,003^{*}$ \\
\hline 17 a 19 anos & 1,00 & & 1,00 & & 1,00 & \\
\hline 20 e 21 anos & $1,16(0,98-1,39)$ & & $1,03(0,95-1,12)$ & & $1,14(0,98-1,33)$ & \\
\hline 22 a 24 anos & $1,08(0,91-1,29)$ & & $0,96(0,88-1,05)$ & & $1,05(0,90-1,23)$ & \\
\hline 25 a 52 anos & $1,21(1,02-1,44)$ & & $0,95(0,87-1,04)$ & & $0,82(0,69-0,98)$ & \\
\hline Situação conjugal ${ }^{\mathrm{b}}$ & & 0,20 & & 0,87 & & 0,003 \\
\hline Sem companheiro(a) & 1,00 & & 1,00 & & 1,00 & \\
\hline Com companheiro(a) & $1,11(0,95-1,30)$ & & $0,99(0,89-1,10)$ & & $0,70(0,56-0,89)$ & \\
\hline Renda pessoal em reais ${ }^{\mathrm{b}}$ & & $0,92^{*}$ & & $0,17^{*}$ & & $0,06^{*}$ \\
\hline Não tem & 1,00 & & 1,00 & & 1,00 & \\
\hline$<\mathrm{R} \$ 510,00$ & $1,09(0,95-1,26)$ & & $0,96(0,90-1,04)$ & & $0,89(0,78-1,01)$ & \\
\hline$\geq \mathrm{R} \$ 510,00$ & $1,01(0,87-1,17)$ & & $0,95(0,88-1,02)$ & & $0,88(0,76-1,01)$ & \\
\hline Escolaridade do pai ${ }^{\mathrm{b}}$ & & $0,003^{*}$ & & $0,83^{*}$ & & $0,03^{*}$ \\
\hline Ensino fundamental incompleto & 1,00 & & 1,00 & & 1,00 & \\
\hline Ensino fundamental completo & $0,81(0,68-0,98)$ & & $1,06(0,96-1,17)$ & & $0,89(0,74-1,07)$ & \\
\hline Ensino médio completo & $0,81(0,70-0,94)$ & & $1,00(0,92-1,09)$ & & $0,93(0,82-1,05)$ & \\
\hline Ensino superior completo & $0,72(0,57-0,91)$ & & $1,00(0,89-1,12)$ & & $0,79(0,66-0,95)$ & \\
\hline Escolaridade da mãe $\mathrm{e}^{\mathrm{b}}$ & & $0,19^{*}$ & & $0,36^{*}$ & & $0,26^{*}$ \\
\hline Ensino fundamental incompleto & 1,00 & & 1,00 & & 1,00 & \\
\hline Ensino fundamental completo & $1,04(0,87-1,25)$ & & $1,04(0,93-1,15)$ & & $0,99(0,80-1,23)$ & \\
\hline Ensino médio completo & $0,98(0,84-1,15)$ & & $0,97(0,90-1,06)$ & & $1,00(0,85-1,17)$ & \\
\hline Ensino superior completo & $0,87(0,69-1,08)$ & & $0,97(0,88-1,07)$ & & $1,13(0,94-1,36)$ & \\
\hline Período de estudo ${ }^{c}$ & & 0,56 & & 0,53 & & 0,25 \\
\hline Diurno & 1,00 & & 1,00 & & 1,00 & \\
\hline Noturno & $1,04(0,91-1,18)$ & & $0,98(0,91-1,05)$ & & $0,92(0,81-1,06)$ & \\
\hline Ano de ingresso $^{c}$ & & $0,42^{*}$ & & $0,71^{*}$ & & $0,02^{*}$ \\
\hline 2010 & 1,00 & & 1,00 & & 1,00 & \\
\hline 2009 & $0,99(0,84-1,17)$ & & $0,99(0,91-1,09)$ & & $1,01(0,85-1,21)$ & \\
\hline 2008 & $1,14(0,97-1,35)$ & & $1,02(0,93-1,12)$ & & $1,26(1,07-1,49)$ & \\
\hline 2007 e anterior & $1,04(0,88-1,23)$ & & $1,01(0,92-1,11)$ & & $1,17(0,98-1,40)$ & \\
\hline $\begin{array}{l}\text { Auto-avaliação dos relacionamentos } \\
\text { com os colegas }^{c}\end{array}$ & & 0,02 & & 0,52 & & 0,32 \\
\hline Positiva & 1,00 & & 1,00 & & 1,00 & \\
\hline Negativa & $1,22(1,03-1,44)$ & & $1,03(0,94-1,14)$ & & $1,09(0,92-1,31)$ & \\
\hline
\end{tabular}

tanto, com relação à escolaridade da mãe $e^{9}$ Considerando a associação entre escolaridade e nível econômico, destaca-se que menores níveis de prática de atividades físicas no lazer são associadas a menor nível econômico ${ }^{29}$, pois, dificulta a obtenção de facilitadores, como a prática em academias e clubes. Outro facilitador para a prática de atividades físicas é o suporte social dos amigos e, neste estudo, os estudantes que avaliaram os relaciona- mentos com os seus colegas de curso como negativo foram mais propensos aos menores níveis de atividades físicas no lazer. A percepção positiva do ambiente acadêmico, como o convívio com os colegas de curso pode ser estimulante à prática, assim como o gosto pela prática de atividades físicas no lazer é fundamental para a adesão ${ }^{24}$.

A maior prevalência de consumo de bebidas alcoólicas foi observada para os homens, o que 
Tabela 4. continuação

\begin{tabular}{|c|c|c|c|c|}
\hline \multirow[t]{2}{*}{ Variáveis } & \multicolumn{2}{|c|}{$\begin{array}{c}\text { Consumo de } \\
\text { bebidas alcoólicas }\end{array}$} & \multicolumn{2}{|c|}{ Fumantes } \\
\hline & RP (IC95\%) & $\mathbf{p}$ & RP (IC95\%) & $\mathbf{p}$ \\
\hline Sexo & & $<0,001$ & & $<0,001$ \\
\hline Masculino & 1,00 & & 1,00 & \\
\hline Feminino & $0,63(0,55-0,73)$ & & $0,20(0,08-0,47)$ & \\
\hline Faixa de idade & & $0,08^{*}$ & & $0,06^{*}$ \\
\hline 17 a 19 anos & 1,00 & & 1,00 & \\
\hline 20 e 21 anos & $1,00(0,79-1,28)$ & & $1,37(0,35-5,37)$ & \\
\hline 22 a 24 anos & $1,14(0,91-1,42)$ & & $1,72(0,48-6,20)$ & \\
\hline 25 a 52 anos & $1,17(0,93-1,45)$ & & $2,72(0,80-9,24)$ & \\
\hline Situação conjugal & & 0,48 & & 0,40 \\
\hline Sem companheiro(a) & 1,00 & & 1,00 & \\
\hline Com companheiro(a) & $0,92(0,72-1,17)$ & & $1,61(0,53-4,92)$ & \\
\hline Renda pessoal em reais & & $0,69^{*}$ & & $0,53^{*}$ \\
\hline Não tem & 1,00 & & 1,00 & \\
\hline$<\mathrm{R} \$ 510,00$ & $0,86(0,71-1,05)$ & & $1,51(0,47-4,85)$ & \\
\hline$\geq \mathrm{R} \$ 510,00$ & $1,03(0,86-1,24)$ & & $1,52(0,44-5,24)$ & \\
\hline Escolaridade do pai & & $0,13^{*}$ & & $0,05^{*}$ \\
\hline Ensino fundamental incompleto & 1,00 & & 1,00 & \\
\hline Ensino fundamental completo & $1,18(0,91-1,54)$ & & $1,61(0,40-6,52)$ & \\
\hline Ensino médio completo & $1,15(0,93-1,43)$ & & $2,26(0,82-6,25)$ & \\
\hline Ensino superior completo & $1,24(0,96-1,60)$ & & $2,84(0,87-9,24)$ & \\
\hline Escolaridade da mãe & & $0,05^{*}$ & & $0,56^{*}$ \\
\hline Ensino fundamental incompleto & 1,00 & & 1,00 & \\
\hline Ensino fundamental completo & $0,98(0,70-1,37)$ & & $1,93(0,37-10,2)$ & \\
\hline Ensino médio completo & $1,16(0,91-1,48)$ & & $1,21(0,32-4,59)$ & \\
\hline Ensino superior completo & $1,28(0,97-1,69)$ & & $1,74(0,43-7,10)$ & \\
\hline Período de estudo & & 0,50 & & 0,21 \\
\hline Diurno & 1,00 & & 1,00 & \\
\hline Noturno & $0,94(0,80-1,12)$ & & $0,56(0,23-1,09)$ & \\
\hline Ano de ingresso & & $0,05^{*}$ & & $0,18^{*}$ \\
\hline 2010 & 1,00 & & 1,00 & \\
\hline 2009 & $0,80(0,63-1,02)$ & & $0,50(0,17-1,52)$ & \\
\hline 2008 & $0,81(0,63-1,04)$ & & $0,26(0,07-0,96)$ & \\
\hline 2007 e anterior & $1,16(0,94-1,44)$ & & $0,47(0,17-1,26)$ & \\
\hline Auto-avaliação dos relacionamentos & & 0,30 & & 0,26 \\
\hline com os colegas & & & 1,00 & \\
\hline Positiva & 1,00 & & $1,71(0,67-4,40)$ & \\
\hline Negativa & $0,86(0,64-1,15)$ & & & \\
\hline
\end{tabular}

RP = Razões de Prevalências; IC95\% = Intervalo de Confiança a 95\%; *Teste de Wald para tendência linear; ${ }^{\ddagger}$ Ajustada para as variáveis do mesmo nível e superior com $\mathrm{p}$ valor do teste de Wald $<0,20 ; \mathrm{a}=1^{\text {a }}$ nível; $\mathrm{b}=2^{\circ}$ nível; $\mathrm{c}=3^{\circ}$ nível.

vai ao encontro do padrão nacional que é caracterizado por maior ocorrência desse comportamento no sexo masculino ${ }^{21,30}$, sendo esta prevalência quase três vezes maior do que a verificada em mulheres ${ }^{21}$. O maior consumo de bebidas alcoólicas, em homens universitários, pode estar associado à ênfase dos meios de comunicação, via propagandas em televisão, jornais/revistas e publicidades nas ruas ${ }^{31}$. Em relação ao tabagismo, somente $3,1 \%$ dos estudantes universitários referiram fumar regularmente, sendo esta a menor prevalência dentre as condutas analisadas, $\mathrm{O}$ que corrobora com outro estudo com estudantes universitários ${ }^{32}$ e também com escolares brasileiros ${ }^{33}$. A menor ocorrência desse comportamento pode estar relacionada à ênfase das políti- 
cas públicas destinadas ao controle e erradicação dessa conduta negativa ${ }^{3}$. Dados de dois inquéritos nacionais mostraram que a prevalência do hábito de fumar em adultos reduziu de 34,8\%, em 1989, para 22,4\% em 2003, sendo a redução mais acentuada entre os homens ${ }^{34}$.

Os resultados não mostraram associação linear entre o ano de ingresso na universidade e o hábito de fumar. Contudo, a menor prevalência de fumantes foi observada entre os estudantes com três anos de vivência acadêmica. Entre os estudantes universitários de Pernambuco não foi verificada associação entre esses indicadores ${ }^{35}$, enquanto que dados de estudantes universitários portugueses mostraram maior prevalência do hábito de fumar naqueles com maior tempo de exposição acadêmica do que nos não expos$\operatorname{tos}^{36}$. A proximidade de conclusão do curso, em período regular de quatro anos, pode representar um importante mediador nos comportamentos relacionados à saúde.

Como limitações deste estudo destacam-se o delineamento transversal que inviabiliza relações de causalidade, além do possível viés de resposta para os comportamentos relacionados à saúde reconhecidos como negativos, como o hábito de fumar, entretanto, foi garantido o sigilo com os dados confidenciais de seleção dos estudantes universitários. O período relativamente curto de coleta de dados minimizou possíveis interferências sazonais, assim como outros vieses de seleção foram evitados mediante o processo de amostragem rigoroso. As informações deste es- tudo representam a linha de base para o acompanhamento das condutas relacionadas à saúde em estudantes universitários, bem como poderá esclarecer os fatores associados às condutas negativas de saúde neste grupo.

As condutas negativas de saúde com maiores prevalências entre os estudantes universitários foram: consumo insuficiente de frutas e de hortaliças e menores níveis de atividades físicas no lazer, seguidas do consumo excessivo de bebidas alcoólicas e hábito de fumar. As maiores prevalências de menores níveis de atividades físicas no lazer foram associadas às mulheres, aos estudantes mais velhos, àqueles com pai com ensino fundamental incompleto e aos que autoavaliaram negativamente os relacionamentos com os colegas do curso. O consumo insuficiente de hortaliças foi maior em homens, estudantes mais jovens, sem companheiro, com pai com o ensino fundamental incompleto e com três anos de exposição acadêmica. $\mathrm{O}$ consumo insuficiente de frutas, o consumo excessivo de bebidas alcoólicas e o hábito de fumar foram associados ao sexo masculino. Estudantes com três anos de vivência acadêmica apresentaram menor prevalência de hábito de fumar. $\mathrm{O}$ incremento de uma alimentação saudável e de práticas de atividades físicas regulares compõe estratégias necessárias para a implementação no contexto universitário, seguindo as da Organização Mundial de Saúde e do Ministério da Saúde do Brasil, visando o controle do aumento da obesidade e de outros agravos crônicos à saúde.

\section{Colaboradores}

TF Sousa desenvolveu e coordena o Estudo MONISA, trabalhou na concepção, análises estatísticas e redação do artigo; HPM José idealizou a proposta do Estudo MONISA e realizou a redação do artigo; AR Barbosa trabalhou na redação e revisão crítica do artigo. 


\section{Referências}

1. World Health Organization (WHO). The world health report 2002. Reducing risks, promoting healthy life. Geneva: WHO; 2002.

2. Yusuf S, Hawken S, Ôunpuu S, Dans T, Avezum A, Lanas F, McQueen M, Budaj A, Pais P, Varigos J, Lisheng L; INTERHEART Study Investigators. Effect of potentially modifiable risk factors associated with myocardial infarction in 52 countries (the INTERHEART study): case-control study. Lancet 2004; 364(9438):937-952.

3. Schmidt MI, Duncan BB, Silva GA, Menezes AM, Monteiro CA, Barreto SM, Chor D, Menezes PR. Chronic non-communicable diseases in Brazil: burden and current challenges. Lancet 2011; 377(9781):1949-1961.

4. Brasil. Secretaria Nacional de Políticas sobre Drogas. I Levantamento Nacional sobre o Uso de Álcool, Tabaco e Outras Drogas entre Universitários das 27 Capitais Brasileiras. Secretaria Nacional de Políticas sobre Drogas; GREA/IPQ-HCFMUSP. Brasília: SENAD; 2010.

5. Wechsler H, Lee JE, Kuo M, Seibring M, Nelson TF, Lee $\mathrm{H}$. Trends in college binge drinking during a period of increased prevention efforts. Findings from 4 Harvard School of Public Health College Alcohol Study Surveys: 1993-2001. J Am Coll Health 2002; 50(5):203-217.

6. Vargas-Zárate M, Becerra-Bulla F, Prieto-Suárez E. Evaluación de la ingesta dietética en estudiantes universitarios. Bogotá, Colombia. Rev Salud Pública 2010; 12(1):116-125.

7. Colares V, Franca C, Gonzalez E. Condutas de saúde entre universitários: diferenças entre gêneros. Cad Saude Publica 2009; 25(3):521-528.

8. Sousa TF. Inatividade física em universitários brasileiros: uma revisão sistemática. Rev Bras Cienc Saúde (IMES) 2011; 9(29):47-55.

9. Silva DAS, Pereira IMM. Estágios de mudança de comportamento para atividade física e fatores associados em acadêmicos de Educação Física. Rev Bras Ativ Fís Saúde 2010; 15(1):15-20.

10. Instituto Nacional de Estudos e Pesquisas Educacionais Anísio Teixeira (INEP). Censo da educação superior. [página na Internet] 2008. [acessado 2010 jul 14]. Disponível em: http://www.inep.gov.br/superior/ censosuperior/default.asp

11. Sousa TF. Atividades físicas praticadas no lazer por universitários de uma instituição pública do Nordeste do Brasil. Rev Bras Ativ Fis Saúde 2012; 17(2): 125-131.

12. Luiz RR, Magnanini MF. A lógica da determinação do tamanho da amostra em investigações epidemiológicas. Cad Saude Coletiva 2000; 8(2):9-28.

13. Sousa TF, Fonseca AS, José HPM, Nahas MV. Validade e reprodutibilidade do questionário Indicadores de Saúde e Qualidade de Vida de Acadêmicos (Isaq-A). Arq Ciên Esporte 2013; 1(1):21-30.
14. United State Department of Health and Human Services. Physical activity and health: a report of the Surgeon General. Atlanta: United State Department of Health and Human Services, Centers for Disease and Prevention, National Center for Chronic Disease Prevention and Health Promotion; 1996.

15. Ainsworth BE, Haskell WL, Whitt MC, Irwin ML, Swarts AM, Strath SJ, O'Brien WL, Bassett DR Jr, Schmitz KH, Emplaincourt PO, Jacobs Jr DR, Leon AS. Compendium of physical activities: an update of activity codes and MET intensities. Med Sci Sports Exerc 2000; 32(9):S498-S516.

16. Jaime PC, Figueiredo ICR, Moura EC, Malta DC. Factors associated with fruit and vegetable consumption in Brazil, 2006. Rev Saude Publica 2009; 43(Supl. 2):57-64.

17. Fonseca SA, Blank VLG, Barros MVG, Nahas MV. Percepção de saúde e fatores associados em industriários de Santa Catarina, Brasil. Cad Saude Publica 2008; 24(3):567-576.

18. Barros MV, Nahas MV. Health risk behaviors, health status self-assessment and stress perception among industrial workers. Rev Saude Publica 2001; 35(6):554-563.

19. Feitosa EPP, Dantas CAO, Andrade-Wartha ERS, Marcellini PS, Mendes-Neto RS. Hábitos alimentares de estudantes de uma universidade pública no Nordeste, Brasil. Alim Nutr 2010; 21(2):225-230.

20. Abolfotouh MA, Bassiouni SA, Mounir GM, Fayyad RC. Health-related lifestyle and risk behaviours among students living in Alexandria University hostels. EMHJ 2007; 13(2):376-391.

21. Brasil. Ministério da Saúde (MS). Vigilância de Fatores de Risco e Proteção para Doenças Crônicas por Inquérito Telefônico: estimativas sobre frequência e distribuição sócio-demográfica de fatores de risco e proteção para doenças crônicas nas capitais dos 26 Estados brasileiros e no Distrito Federal em 2007. Brasília: MS; 2008.

22. Pino LA, Cediel GG, Hirsch BS. Ingesta de alimentos de origen animal versus origen vegetal y riesgo cardiovascular. Rev Chil Nutr 2009; 36(3):210-216.

23. Löllgen H, Böckenhoff A, Knapp G. Physical activity and all-cause mortality: an updated meta-analysis with different intensity categories. Int J Sports Med 2009; 30(3):213-224.

24. Mielke GI, Ramis TR, Habeyche EC, Oliz MM, Tessmer S, Azevedo MR, Hallal PC. Atividade física e fatores associados em universitários do primeiro ano da Universidade Federal de Pelotas. Rev Bras Ativ Fís Saúde 2010; 15(1):57-64.

25. Silva DAS, Petroski EL. Fatores associados ao nível de participação em atividades físicas em estudantes de uma universidade pública no sul do Brasil. Cien Saude Colet 2011; 16(10):4087-4094. 
26. Haase A, Steptoe A, Sallis JF, Wardle J. Leisuretime physical activity in university students from 23 countries: associations with health beliefs, risk awareness, and national economic development. Prev Med 2004; 39(1):182-190.

27. Silva DAS, Petroski EL, Reis RS. Barreiras e facilitadores de atividades físicas em frequentadores de parques públicos. Motriz: rev educ fis 2009; 15(2):219227.

28. Sousa TF, Santos SFS, José HPM. Barreiras percebidas à prática de atividade física no Nordeste do Brasil. Pensar a Prática 2010; 13(1):1-15.

29. Azevedo MR, Horta BL, Gigante DP, Victora CG, Barros FC. Factors associated to leisure-time sedentary lifestyle in adults of 1982 birth cohort, Pelotas, Southern Brazil. Rev Saude Publica 2008; 42(Supl. 2):1-7.

30. Almeida-Filho N, Lessa I, Magalhães L, Araújo MJ, Aquino E, Kawachi I, James SA. Determinantes sociais e padrões de consumo de álcool na Bahia, Brasil. Rev Saude Publica 2004; 38(1):45-54.

31. Pedrosa AAS, Camacho LAB, Passos SRL, Oliveira RVC. Consumo de álcool entre estudantes universitários. Cad Saude Publica 2011; 27(8):1611-1621.

32. Granville-Garcia AF, Sarmento DJS, Santos JA, Pinto TA, Sousa RV, Cavalcanti AL. Tabagismo entre acadêmicos da área de saúde. Cien Saude Colet 2012; 17(2):389-396.

33. Malta DC, Sardinha LMV, Mendes I, Barreto SM, Giatti L, Castro IRR, Moura Ld, Dias AJ, Crespo C. Prevalência de fatores de risco e proteção de doenças crônicas não transmissíveis em adolescentes: resultados da Pesquisa Nacional de Saúde do Escolar (PeNSE), Brasil, 2009. Cienc Saude Colet 2010; 15(Supl. 2):3009-3019.

34. Monteiro CA, Cavalcante TM, Moura EC, Claro RM, Szwarcwald CL. Population-based evidence of a strong decline in the prevalence of smokers in Brazil (1989-2003). Bull World Health Organ 2007; 85(7):527-534.

35. Franca C, Colares V. Estudo comparativo de condutas de saúde entre universitários no início e no final do curso. Rev Saude Publica 2008; 42(3):420427.

26. Brandão MP, Pimentel FL, Cardoso MF. Impact of academic exposure on health status of university students. Rev Saude Publica 2011; 45(1):49-58.

Artigo apresentado em 17/04/2012

Aprovado em 23/05/2012

Versão final apresentada em 06/06/2012 
